\title{
ANTIPODAL MANIFOLDS IN COMPACT SYMMETRIC SPACES OF RANK ONE
}

\author{
JUAN ALFREDO TIRAO
}

\begin{abstract}
Let $M$ be a compact Riemannian globally symmetric space of rank one. A theorem due to Helgason states that the antipodal manifold $A_{x}$ of a point $x \in M$ is again a symmetric space of rank one. We compute the multiplicities of the restricted roots of $A_{x}$ from those of $M$, obtaining a very convenient way to determine $A_{x}$.
\end{abstract}

1. Introduction. Let $M$ be a compact Riemannian globally symmetric space of rank one. Let $L$ denote the diameter of $M$ and if $x \in M$ let $A_{x}$ denote the corresponding antipodal manifold, that is the set of points $y \in M$ at distance $L$ from $x ; A_{x}$ is indeed a manifold and with the Riemannian structure induced by $M$, is a symmetric space of rank one totally geodesic in $M$ (see Theorem 2.5). Associated to $M$ there is a triple of numbers $(p, q, \lambda)$ (see below for the definition) which appear in different places, for example in the expressions, in geodesic polar coordinates, of the Riemannian measure and the Laplace-Beltrami operator of $M$. Sometimes doing analysis on these spaces it is necessary to know $p_{1}, q_{1}$ and $\lambda_{1}$, the values of $p, q$ and $\lambda$ for an antipodal manifold of $M$ (see [2] and [4]). The complete list of compact Riemannian globally symmetric spaces of rank one, with the corresponding values of $p, q$ and $\lambda$ is (see [4, p. 171]):

The spheres $\mathbf{S}^{n}, n=1,2, \ldots: p=0, q=n-1, \lambda=\pi / 2 L$,

The real projective spaces $\mathbf{P}^{n}(\mathbf{R}), n=2,3, \ldots: p=0, q=n-1, \lambda=$ $\pi / 4 L$,

The complex projective spaces $\mathbf{P}^{n}(\mathbf{C}), n=4,6, \ldots: p=n-2, q=1$, $\lambda=\pi / 2 L$,

The quaternion projective spaces $\mathbf{P}^{n}(\mathbf{H}), n=8,12, \ldots: p=n-4, q=3$, $\lambda=\pi / 2 L$,

The Cayley projective space $\mathbf{P}^{16}($ Cay): $p=8, p=7, \lambda=\pi / 2 L$.

The superscripts denote the real dimension. The corresponding antipodal manifolds are also known ([1, pp. 437-467], [5, pp. 35 and 52]), but the computations involved are not simple. In this paper we compute $p_{1}, q_{1}$ and $\lambda_{1}$ directly from $p, q$ and $\lambda$. Since the triple $(p, q, \lambda)$ characterizes $M$, we also obtain a very convenient way to determine the antipodal manifolds of $M$.

Received by the editors October 20, 1977.

AMS (MOS) subject classifications (1970). Primary 53C35.

Key words and phrases. Geodesic symmetry, semisimple Lie group, involutive automorphism, restricted root, projective spaces. 
2. We assume $\operatorname{dim} M>1$. This is the case which interests us, and it has the convenient implication that the group $I(M)$ of isometries of $M$, in the compact open topology, is semisimple. Let $o$ be a fixed point in $M$ and $s_{o}$ the geodesic symmetry of $M$ with respect to $o$. Let $U$ denote the identity component of $I(M), \mathfrak{u}$ the Lie algebra of $U$ and $\mathfrak{u}=\mathfrak{l}+\mathfrak{p}$ the decomposition of $\mathfrak{u}$ into eigenspaces of the involutive automorphism $d \gamma$ of $\mathfrak{u}$ which corresponds to the automorphism $\gamma: u \mapsto s_{o} u s_{o}$ of $U$. Here $f$ is the Lie algebra of the subgroup $K$ of $U$ which leaves $o$ fixed. Changing the distance function $d$ on $M$ by a constant factor we may assume that the differential of the mapping $\pi$ : $u \mapsto u \cdot o$ of $U$ onto $M$ gives an isometry of $\mathfrak{p}$ (with the metric of the negative of the Killing form of $u$ ) onto $M_{o}$, the tangent space to $M$ at $o$.

Let $X \mapsto \operatorname{ad}(X)$ denote the adjoint representation of $\mathfrak{u}$. Select a vector $H \in \mathfrak{p}$ of length $L$. The space $\mathfrak{a}=\mathbf{R} H$ is a Cartan subalgebra of the symmetric space $M$ and we can select a positive restricted root $\alpha$ of $M$ such that $\frac{1}{2} \alpha$ is the only other possible positive restricted root. This means that the eigenvalues of $(\operatorname{ad} H)^{2}$ are $0, \alpha(H)$ and possibly $\left(\frac{1}{2} \alpha(H)\right)^{2} ; \alpha(H)$ is purely imaginary. Let $\mathfrak{u}=\mathfrak{u}_{0}+\mathfrak{u}_{\alpha}+\mathfrak{u}_{\alpha / 2}$ be the corresponding decomposition of $\mathfrak{u}$ into eigenspaces and $\mathfrak{f}_{\beta}=\mathfrak{u}_{\beta} \cap \mathfrak{f}, \mathfrak{p}_{\beta}=\mathfrak{u}_{\beta} \cap \mathfrak{p}$ for $\beta=0, \alpha, \frac{1}{2} \alpha$. Then $\mathfrak{p}_{0}=\mathfrak{a}$ and $\mathfrak{f}_{\beta}=\operatorname{ad} H\left(\mathfrak{p}_{\beta}\right)$ for $\beta \neq 0$. We let $p=\operatorname{dim} \mathfrak{p}_{\alpha / 2}, q=\operatorname{dim} \mathfrak{p}_{\alpha}$ and $\lambda=|\alpha(H)| / 2 L$.

The geodesics in $M$ are all closed and have length $2 L$ and the exponential mapping Exp at $o$ is a diffeomorphism of the open ball in $M$ of center 0 and radius $L$ onto the complement $M-A_{o}$ (see [3, Chapter IX, §5]).

LEMMA 2.1. (i) $\alpha(H)= \pm \pi i$ if $\frac{1}{2} \alpha$ is a restricted root; (ii) $\alpha(H)= \pm \pi i / 2$ if $H$ is not conjugate to 0 ; (iii) $\alpha(H)= \pm \pi i$ if $\frac{1}{2} \alpha$ is not a restricted root and $H$ is conjugate to 0 .

Proof. Considering Exp as a map of $\mathfrak{p}$ onto $M$ we have that $\operatorname{Exp} X=o$ for all $X \in \mathfrak{p}$ of length $2 L$. Hence $d \operatorname{Exp}_{2 H}$ vanishes identically on the orthogonal complement of $a$ in $\mathfrak{p}$. This orthogonal complement is precisely $\mathfrak{p}_{\alpha}+\mathfrak{p}_{\alpha / 2}$. Using the formula for $d \operatorname{Exp}_{2 H}$ [3, Theorem 4.1] it follows that $\alpha(2 H) \in \pi i \mathbf{Z}$, and $\frac{1}{2} \alpha(2 H) \in \pi i \mathrm{Z}$ when $\frac{1}{2} \alpha$ is a restricted root. Thus if $\frac{1}{2} \alpha$ is a restricted root $\alpha(H)=n \pi i$. We also have $|n|=1$ because otherwise $n^{-1} H$ would be conjugate to 0 and of length less than $L$, which is impossible. This proves (i). To prove (ii) we observe that $\alpha\left(2 n^{-1} H\right)=\pi i$ for some $n \in \mathbf{Z}$. As before it follows that $|n| \leqslant 2$. Now the assumption that $H$ is not conjugate to 0 implies $|n|=1$. For (iii) we also have $|n| \leqslant 2$. But now $|n|=2$ because $H$ is conjugate to 0 . Q.E.D.

It will be convenient to choose $H$ so that the above lemma holds with the minus sign. Let $o_{1}$ denote the point $\operatorname{Exp}(-H)$.

Proposition 2.2. Let $G$ denote the subgroup of $U$ leaving the point $o_{1} \in M$ fixed. Then $\gamma(G)=G$. 
Proof. We have $G=\exp (-H) K \exp H$ because $\exp (-H) \cdot o=o_{1}$. If $g=\exp (-H) k \exp H, k \in K$, then $\gamma(g)=\exp H k \exp (-H)$. Hence to prove the assertion it suffices to show that $\exp H \cdot o=o_{1}$. We have $\operatorname{Exp}(t+$ 2) $H=\operatorname{Exp} t H$ for all $t \in \mathbf{R}$, therefore

$$
\exp H \cdot o=\operatorname{Exp} H=\operatorname{Exp}(-H)=o_{1} . \quad \text { Q.E.D. }
$$

Corollary. 2.3. Let $U^{1}$ denote the identity component of $G$ and $K^{1}=U^{1} \cap$ $K$. Then $\left(U^{1}, K^{1}\right)$ is a Riemannian symmetric pair.

Proof. It is a enough to check that $\left(U_{\gamma}^{1}\right)_{0} \subset K^{1} \subset U_{\gamma}^{1}$, where $U_{\gamma}^{1}$ is the set of fixed points of $\gamma$ in $U^{1}$ and $\left(U_{\gamma}^{1}\right)_{0}$ is the identity component of $U_{\gamma}^{1}$. If $u \in\left(U_{\gamma}^{1}\right)_{0}$ then $u \in\left(U_{\gamma}\right)_{0}$, hence $u \in K$ because $(U, K)$ is a symmetric pai [3, Theorem 3.3]. The other inclusion follows similarly. Q.E.D.

Let $\mathfrak{u}^{1}$ denote the Lie algebra of $U^{1}$ and let $\mathfrak{u}^{1}=\mathfrak{f}^{1}+\mathfrak{p}^{1}$ be the decomposition of $\mathfrak{u}^{1}$ into eigenspaces of the involutive automorphism of $\mathfrak{u}^{1}$ which corresponds to the automorphism $\gamma: U^{1} \rightarrow U^{1}$.

Proposition 2.4. (i) When $\frac{1}{2} \alpha$ is a restricted root we have

$$
\mathfrak{f}^{1}=\mathfrak{f}_{0}+\mathfrak{f}_{\alpha}, \quad \mathfrak{p}^{1}=\mathfrak{p}_{\alpha / 2} .
$$

(ii) If $H$ is not conjugate to 0

$$
\mathfrak{f}^{1}=\mathfrak{l}_{0}, \quad \mathfrak{p}^{1}=\mathfrak{p}_{\alpha} .
$$

(iii) If $\frac{1}{2} \alpha$ is not a restricted root and $H$ is conjugate to 0 , then

$$
\mathfrak{f}^{1}=\mathfrak{f}_{0}+\mathfrak{l}_{\alpha}, \quad \mathfrak{p}^{1}=\{0\} .
$$

Proof. The Lie algebra of $U^{1}$ is given by

$$
\mathfrak{u}^{1}=\left\{X \in \mathfrak{u}: \exp (t X) o_{1}=o_{1} \text { for all } t \in \mathbf{R}\right\} \text {. }
$$

Therefore $X \in \mathfrak{u}^{1}$ if and only if $\exp (t X) \exp (-H) o=\exp (-H) o$ for all $t \in \mathbf{R}$, which is equivalent to

$$
\exp (\operatorname{Ad}(\exp H) t X) \in K \quad \text { all } t \in \mathbf{R},
$$

where Ad denotes the adjoint representation of $U$. Hence

$$
\mathfrak{u}^{1}=\{X \in \mathfrak{u}: \operatorname{Ad}(\exp H) X \in \mathfrak{f}\} .
$$

Let $X_{\beta}$ be a vector in $\mathfrak{u}_{\beta}$ for $\beta=0, \alpha, \frac{1}{2} \alpha$. A direct computation which is left to the reader, yields

$$
\begin{gathered}
\operatorname{Ad}(\exp H)\left(X_{0}+X_{\alpha}+X_{\alpha / 2}\right)=X_{0}+\cosh (\alpha(H)) X_{\alpha}+\cosh \left(\frac{1}{2} \alpha(H)\right) X_{\alpha / 2} \\
+\alpha(H)^{-1} \text { ad } H\left(\sinh (\alpha(H)) X_{\alpha}+2 \sinh \left(\frac{1}{2} \alpha(H)\right) X_{\alpha / 2}\right) .
\end{gathered}
$$

Now the proposition follows from (2.1) and Lemma 2.1 by simple inspection. Q.E.D.

Theorem 2.5. (Cf. [4] Proposition 5.1.) When $\frac{1}{2} \alpha$ is a restricted root or $H$ is not conjugate to 0 the orbit $M_{1}=U^{1} \cdot o$, with the Riemannian structure induced 
by $M$, is a symmetric space of rank one and a totally geodesic submanifold of $M$.

When $\frac{1}{2} \alpha$ is not a restricted root and $H$ is conjugate to 0 the orbit $M_{1}=U^{1} \cdot o$ reduces to a point $\{o\}$. In both cases $M_{1}=A_{o_{1}}$.

Proof. Let $y \in M_{1}$. Writing $y=u \cdot o, u \in U^{1}$, we have $s_{y}(v \cdot o)=$ $u \gamma\left(u^{-1} v\right) \cdot o \in M_{1}$ for $v \in U^{1}$ (see Proposition 2.2); hence $s_{y}\left(M_{1}\right)=M_{1}$. If $\gamma_{y}$ denotes the restriction of $s_{y}$ to $M_{1}$, then $\gamma_{y}$ is an involutive isometry of $M_{1}$ with $y$ as isolated fixed point. Thus $M_{1}$ is globally symmetric and $\gamma_{y}$ is the geodesic symmetry with respect to $y$.

To prove that $M_{1}$ is totally geodesic it suffices to show that each $M$-geodesic which is tangent to $M_{1}$ at $o$ is a path in $M_{1}$. Let $t \rightarrow \operatorname{Exp} t X, t \in \mathbf{R}$, be one of such geodesics. Then $X \in \mathfrak{p}^{1}=\mathfrak{p} \cap \mathfrak{u}^{1}$. Hence $\operatorname{Exp} t X=\exp (t X) \cdot o \in$ $M_{1}, t \in \mathbf{R}$, and our geodesic is a path in $M$. Consequently, the first two assertions follow from the definition of rank and Proposition 2.4.

Clearly, $M_{1} \subset A_{o_{1}}$, both submanifolds are connected, and of the same dimension since $A_{o}=K \cdot o_{1}$. Hence $M_{1}=A_{o_{1}}$. This completes the proof of the theorem. Q.E.D.

Proposition 2.6. Let $N$ be the kernel of the restriction homomorphism $U^{1} \rightarrow I\left(M_{1}\right)$. Then $U^{1} / N$ is naturally isomorphic to the identity component $I_{0}\left(M_{1}\right)$ of $I\left(M_{1}\right)$.

Proof. If $\operatorname{dim} M_{1} \leqslant 1$ then $M_{1}$ is either a point or $S^{1}$ (circle) and $\operatorname{dim} I_{0}\left(M_{1}\right) \leqslant 1$. The proposition is obviously true in these cases. Therefore we now assume $\operatorname{dim} M_{1}>1$.

Let $z$ be the center of $\mathfrak{u}^{1}$, then $z=\left(z \cap \mathfrak{f l}^{1}\right)+\left(z \cap \mathfrak{p}^{1}\right)$. But $z \cap \mathfrak{p}^{1}=\{0\}$ because $M_{1}$ is a symmetric space of rank one totally geodesic in $M$. Thus $f^{1} \supset z$ and $K^{1} \supset Z_{0}$, the identity component of the center $Z$ of $U^{1}$. Now $Z_{0} \subset Z \subset K^{1} \subset N$. Hence $z \subset \mathfrak{n}$, the Lie algebra of $N$. Therefore we have a surjective Lie algebra homomorphism $\mathfrak{u}^{1} / \mathfrak{z} \rightarrow \mathfrak{u}^{1} / \mathfrak{n}$, which implies thar $U^{1} / N$ is semisimple since $\mathfrak{u}^{1} / \mathfrak{z}$ is semisimple.

We also have $N \subset K^{1}$, and we want to consider the pair $\left(U^{1} / N, K^{1} / N\right)$. Given $u \in N$ and $v \in U^{1}$ we have $\gamma(u)(v \cdot o)=s_{o} u(\gamma(v) \cdot o)=s_{o} \gamma(v) \cdot o=$ $v \cdot o$ (see Proposition 2.2), hence $\gamma(N) \subset N$. Therefore $\gamma$ induces an involutive analytic automorphism, denoted also by $\gamma$, of $U^{1} / N$. We shall prove that $\gamma$ turns $\left(U^{1} / N, K^{1} / N\right)$ into a symmetric pair. In fact, $K^{1} / N \subset U_{\gamma}^{1} / N \subset$ $\left(U^{1} / N\right)_{\gamma}$. On the other hand, if $\exp (t X) N \in\left(U^{1} / N\right)_{\gamma}, X \in \mathfrak{u}^{1}$, then $\exp (-t X) \gamma(\exp t X) \in N$. Thus $-X+d \gamma(X) \in \mathrm{n} \subset \mathfrak{f}^{1}$, which implies $X \in$ $\mathfrak{f}^{1}$. Therefore $\left(\left(U^{1} / N\right)_{\gamma}\right)_{0} \subset K^{1} / N$.

At this point the proposition follows as a consequence of $[3$, Theorem 4.1]. Q.E.D.

When $I_{0}\left(M_{1}\right)$ is identified with $U^{1} / N$ the isotropy subgroup of $I_{0}\left(M_{1}\right)$ at $o \in M_{1}$ becomes $K^{1} / N$ and the decomposition of the Lie algebra of $I_{0}\left(M_{1}\right)$ under the corresponding involutive automorphism can be written as $\mathfrak{u}^{1} / \mathfrak{n}=$ $\mathfrak{f}^{1} / \mathfrak{n}+\mathfrak{p}^{1}$. Therefore the constants $p_{1}, q_{1}$ and $\lambda_{1}$ associated to $M_{1}$ can be 
computed from $\left(\operatorname{ad} H_{1}\right)^{2}: \mathfrak{p}^{1} \rightarrow \mathfrak{p}^{1}$, where $H_{1} \in \mathfrak{p}^{1}$ is a vector of length $L$ ( $L$ is also the diameter of $M_{1}$, see Theorem 2.5). In particular we obtain $\lambda_{1}=\lambda$.

Proposition 2.7. If $H$ is not conjugate to $0, p_{1}=0$ and $q_{1}=q-1$.

Proof. Since $H$ and $H_{1}$ are conjugate under $K,(\operatorname{ad} H)^{2}$ and $\left(\operatorname{ad} H_{1}\right)^{2}$, as linear transformations of $\mathfrak{p}$, have the same eigenvalues with the same multiplicities. These eigenvalues are 0 and $\alpha(H)^{2}$ (see Lemma 2.1) with multiplicities 1 and $q$, respectively. Now the proposition follows because 0 is an eigenvalue of $\left(\operatorname{ad} H_{1}\right)^{2}: \mathfrak{p}^{1} \rightarrow \mathfrak{p}^{1}$ and $\operatorname{dim} \mathfrak{p}^{1}=\operatorname{dim} \mathfrak{p}_{\alpha}=q$ (see Proposition 2.4). Q.E.D.

From now on we shall assume that $\frac{1}{2} \alpha$ is a restricted root.

Let $\mathfrak{u}_{\mathbf{C}}$ be the complexification of $\mathfrak{u}, \theta$ the corresponding extension of $d \gamma$ and $B$ the Killing form of $\mathfrak{u}_{\mathbf{c}}$. Let $\mathfrak{h}$ be any maximal abelian subalgebra of $\mathfrak{u}$ containing $\mathfrak{a}$ and let $\mathfrak{h}_{\mathbf{C}}, \mathfrak{a}_{\mathbf{C}}$ and $\mathfrak{p}_{\mathbf{C}}$ denote the subspaces of $\mathfrak{u}_{\mathbf{C}}$ generated by $\mathfrak{h}$, $\mathfrak{a}$ and $\mathfrak{p}$, respectively. Then $\mathfrak{h}_{\mathbf{c}}$ is a Cartan subalgebra of $\mathfrak{u}_{\mathbf{c}}$. Now select compatible orderings in the dual spaces of $i$ and $i \mathfrak{h}$, respectively. Let $\Delta$ denote the set of all nonzero roots (of $\mathfrak{u}_{\mathbf{C}}$ with respect to $\mathfrak{h}_{\mathbf{C}}$ ) and let $\Delta^{+}$ denote the set of all positive roots. Now for each $\lambda \in \Delta$ the linear function $\lambda^{\theta}$ defined by $\lambda^{\theta}(X)=\lambda(\theta X), X \in \mathfrak{h}_{\mathbf{C}}$, is again a member of $\Delta$ and $\lambda(H)$ is either 0 , or $\pm \frac{1}{2} \alpha(H)$, or $\pm \alpha(H)$ when $H \in a$. We may assume that $\lambda(H)$ is equal to $\beta(H)\left(\beta=0, \alpha\right.$ or $\left.\frac{1}{2} \alpha\right)$ whenever $\lambda \in \Delta^{+}, H \in$ a being the vector already chosen.

LEMMA 2.8. For each $\lambda \in \Delta^{+}$such that $\lambda(H)=\beta(H), \beta \neq 0$, select $a$ nonzero vector $X_{\lambda}$ in the corresponding root subspace. Then the $X_{\lambda}-\theta X_{\lambda}$ 's form a basis of $\mathfrak{p}_{\beta}+i \mathfrak{p}_{\beta}$.

Proof. Clearly the vectors $X_{\lambda}$ 's, $\tau X_{\lambda}$ 's form a basis of $\mathfrak{u}_{\beta}+i \mathfrak{u}_{\beta}$, $\tau$ being the conjugation of $\mathfrak{u}_{\mathbf{C}}$ with respect to $\mathfrak{u}$. When $\lambda$ runs over the set of all positive roots such that $\lambda(H)=\beta(H), \beta \neq 0, \tau X_{\lambda}$ runs over all root subspaces corresponding to all negative roots $\mu$ such that $\mu(H)=-\beta(H)$. But this is precisely what happens with the vectors $\theta X_{\lambda}$. Thus the vectors $X_{\lambda}$ 's and $\theta X_{\lambda}$ 's form also a basis of $\mathfrak{u}_{\beta}+i \mathfrak{u}_{\beta}$. Now the assertion is clear. Q.E.D.

LEMma 2.9. For all $Y \in \mathfrak{p}_{\beta}+i \mathfrak{p}_{\beta}, \beta \neq 0$, we have that $(\operatorname{ad} Y)^{2} a_{\mathbf{c}} \subset a_{\mathbf{c}}$.

Proof. Let $\lambda, \mu \in \Delta^{+}$such that $\lambda(H)=\mu(H)$. By the preceding lemma it suffices to prove that

$$
\left(\operatorname{ad}\left(X_{\lambda}-\theta X_{\lambda}\right) \operatorname{ad}\left(X_{\mu}-\theta X_{\mu}\right)+\operatorname{ad}\left(X_{\mu}-\theta X_{\mu}\right) \operatorname{ad}\left(X_{\lambda}-\theta X_{\lambda}\right)\right) H \in a_{\mathbf{C}} .
$$

We have

$$
\left[X_{\mu}-\theta X_{\mu}, H\right]=-\mu(H) X_{\mu}+\mu^{\theta}(H) \theta X_{\mu}=-\mu(H)\left(X_{\mu}+\theta X_{\mu}\right),
$$

and 


$$
\begin{aligned}
\operatorname{ad}\left(X_{\lambda}-\theta X_{\lambda}\right) \operatorname{ad}\left(X_{\mu}-\theta X_{\mu}\right) H & =-\mu(H)\left[X_{\lambda}-\theta X_{\lambda}, X_{\mu}+\theta X_{\mu}\right] \\
= & -\mu(H)\left(\left[X_{\lambda}, X_{\mu}\right]+\left[X_{\lambda}, \theta X_{\mu}\right]-\left[\theta X_{\lambda}, X_{\mu}\right]-\theta\left[X_{\lambda}, X_{\mu}\right]\right) .
\end{aligned}
$$

Interchanging $\lambda$ and $\mu$ and adding we obtain

$$
\begin{gathered}
\left(\operatorname{ad}\left(X_{\lambda}-\theta X_{\lambda}\right) \operatorname{ad}\left(X_{\mu}-\theta X_{\mu}\right)+\operatorname{ad}\left(X_{\mu}-\theta X_{\mu}\right) \operatorname{ad}\left(X_{\lambda}-\theta X_{\lambda}\right)\right) H \\
=-2 \lambda(H)\left(\left[X_{\lambda}, \theta X_{\mu}\right]-\left[\theta X_{\lambda}, X_{\mu}\right]\right) .
\end{gathered}
$$

Now

$$
\left[H,\left[X_{\lambda}, \theta X_{\mu}\right]\right]=\left(\lambda(H)+\mu^{\theta}(H)\right)\left[X_{\lambda}, \theta X_{\mu}\right]=0
$$

and also $\left[H,\left[\theta X_{\lambda}, X_{\mu}\right]\right]=0$. But $\left[X_{\lambda}, \theta X_{\mu}\right]-\left[\theta X_{\lambda}, X_{\mu}\right] \in \mathfrak{p}_{\mathbf{C}}$, hence it belongs to $a_{\mathbf{C}}$, in view of the maximality of $a_{\mathbf{C}}$. This proves our assertion and hence the lemma. Q.E.D.

Note that Lemma 2.9 holds even when $\operatorname{rank}(\mathfrak{u})>1$.

Proposition 2.10. Let $q(Y)$ be the complex number defined by $(\operatorname{ad} Y)^{2} H=$ $q(Y) H, Y \in \mathfrak{p}_{\alpha / 2}+i \mathfrak{p}_{\alpha / 2}$. Then $q(Y)=(\pi / 2 L)^{2} B(Y, Y)$ and $(\operatorname{ad} Y)^{2} Z=$ $q(Y) Z$ for all $Z \in a+\mathfrak{p}_{\alpha}$.

Proof. It is enough to consider $Y \in \mathfrak{p}_{\alpha / 2}$. In fact, $q$ is a quadratic form on $\mathfrak{p}_{\alpha / 2}+i \mathfrak{p}_{\alpha / 2}$, since

$\left(\operatorname{ad}\left(Y_{1}+Y_{2}\right)^{2}-\operatorname{ad}\left(Y_{1}\right)^{2}-\operatorname{ad}\left(Y_{2}\right)^{2}\right) H=\left(\operatorname{ad} Y_{1} \operatorname{ad} Y_{2}+\operatorname{ad} Y_{2} \operatorname{ad} Y_{1}\right) H$

The identity component $K_{0}^{1}$ of $K^{1}$ acts transitively on any sphere in $\mathfrak{a}+\mathfrak{p}_{\alpha}$ with center 0 . In fact, $a+\mathfrak{p}_{\alpha}$ is orthogonal to $\mathfrak{p}_{\alpha / 2}$ and therefore also stable under $K^{1}$. Moreover, the tangent space to the orbit $\operatorname{Ad}\left(K_{0}^{1}\right) H$ at the point $H$ is $\left[\mathfrak{f}^{1}\right.$, a] which equals $\mathfrak{p}_{\alpha}$ (see Proposition 2.4(i)). It follows that $\operatorname{Ad}\left(K_{0}^{1}\right) H$ is the sphere in $a+\mathfrak{p}_{\alpha}$ of radius $L$ and center 0 .

Now take $x \in K_{0}^{1}, Y \in \mathfrak{p}_{\alpha / 2}$, then

$$
\operatorname{ad}(\operatorname{Ad}(x) Y)^{2}(\operatorname{Ad}(x) H)=\operatorname{Ad}(x)(\operatorname{ad} Y)^{2} H=q(Y) \operatorname{Ad}(x) H .
$$

This shows that $(\operatorname{ad} Y)^{2}$ acts as a scalar transformation on $a+\mathfrak{p}_{\alpha}$, multiplying by $q(Y)$.

If $Y$ has length $L$ the eigenvalues of $(\operatorname{ad} Y)^{2}$ as a linear transformation of $\mathfrak{p}$ are the same as those of $(\operatorname{ad} H)^{2}$. Thus $q(Y)=\left(\frac{1}{2} \alpha(H)\right)^{2}$ since $\operatorname{dim}\left(\mathfrak{a}+\mathfrak{p}_{\alpha}\right)$ $=q+1$. From Lemma 2.1 we get $\left(\frac{1}{2} \alpha(H)\right)^{2}=-(\pi / 2)^{2}$, from where the proposition follows by homogeneity. Q.E.D.

THEOREM 2.11. If $\frac{1}{2} \alpha$ is a restricted root then $p_{1}=p-q-1$ and $q_{1}=q$.

Proof. Given $H_{1} \in \mathfrak{p}^{1}=\mathfrak{p}_{\alpha / 2}$ of length $L$ the eigenvalues of $\left(\operatorname{ad} H_{1}\right)^{2}$ in $\mathfrak{p}$ are $0,-(\pi / 2)^{2}$ and $-\pi^{2}$ with multiplicities $1, p$ and $q$, respectively. Proposition 2.10 says that $\left(\operatorname{ad} H_{1}\right)^{2}$ on $\mathfrak{a}+\mathfrak{p}_{\alpha}$ has $-(\pi / 2)^{2}$ as an eigenvalue of multiplicity $1+q$. Therefore the eigenvalues of $\left(\operatorname{ad} H_{1}\right)^{2}$ in $\mathfrak{p}_{\alpha / 2}$ are 0 , $-(\pi / 2)^{2}$ and $-\pi^{2}$ with multiplicities $1, p-q-1$ and $q$, respectively. Q.E.D. 
COROllaRY 2.12. The spheres $\mathbf{S}^{n}(n=1,2, \ldots)$, the real projective spaces $\mathbf{P}^{n}(\mathbf{R}),(n=2,3, \ldots)$, the complex projective spaces $\mathbf{P}^{n}(\mathbf{C})(n=4,6, \ldots)$, the quaternion projective spaces $\mathbf{P}^{n}(\mathbf{H})(n=8,12, \ldots)$ and the Cayley projective plane $\mathbf{P}^{16}$ (Cay) are all the Riemannian globally symmetric spaces of rank 1 . The superscripts denote the real dimension. The corresponding antipodal manifolds are in the respective cases: $A$ point, $\mathbf{P}^{n-1}(\mathbf{R}), \mathbf{P}^{n-2}(\mathbf{C}), \mathbf{P}^{n-4}(\mathbf{H}), \mathbf{S}^{8}$.

\section{REFERENCES}

1. E. Cartan, Sur certaines formes riemannienes remarquables des géométries a groupe fondamental simple, Ann. Sci. Ecole Norm. Sup. 44 (1927), 345-467.

2. R. Gandulfo and J. Tirao, Multiplier transformations of functions on compact two-point homogeneous spaces, Trabalhos de Matemática 126 (1977).

3. S. Helgason, Differential geometry and symmetric spaces, Academic Press, New York, 1962.

4. __ The Radon transform on Euclidean spaces, compact two-point homogeneous spaces and Grassmann manifolds, Acta Math. 113 (1965), 153-180.

5. T. Nagano, Homogeneous sphere bundles and the isotropic Riemannian manifolds, Nagoya Math. J. 15 (1959), 29-55.

Instituto de Matematica, Astronoma y Fisica, Universidad Nacional de Cordoba, Cordoba, Argentina 